\title{
Establishing Better Evidence on Remote Monitoring for Postpartum Hypertension: A Silver Lining of the Coronavirus Pandemic
}

\author{
Michala R. Sawyer, BA ${ }^{1,2}$ Elana F. Jaffe, BA ${ }^{3}$ Mariam Naqvi, MD 4 Amy Sarma, MD 5,6 \\ William H. Barth Jr., MD ${ }^{1,2}$ Ilona T. Goldfarb, MD, $\mathrm{MPH}^{1,2}$
}

${ }^{1}$ Division of Maternal-Fetal Medicine, Department of Obstetrics and Gynecology, Massachusetts General Hospital, Boston, Massachusetts

2 Division of Maternal-Fetal Medicine, Department of Obstetrics and Gynecology, Harvard Medical School, Boston, Massachusetts

${ }^{3}$ Department of Maternal, Child, and Family Health, Department of Social Medicine, Center for Bioethics, Gillings School of Global Public Health, The University of North Carolina at Chapel Hill, Chapel Hill, North Carolina

${ }^{4}$ Division of Maternal-Fetal Medicine, Department of Obstetrics and Gynecology, Cedars-Sinai Medical Center, Los Angeles, California

${ }^{5}$ Division of Cardiology, Department of Medicine, Massachusetts General Hospital, Boston, Massachusetts

${ }^{6}$ Division of Cardiology, Department of Medicine, Harvard Medical School, Boston, Massachusetts

\author{
Address for correspondence Ilona Goldfarb, MD, MPH, Department \\ of Obstetrics and Gynecology, Massachusetts General Hospital, \\ 55 Fruit Street - Founders 4, Boston, MA 02114 \\ (e-mail: igoldfarb@partners.org).
}

Am J Perinatol Rep 2020;10:e315-e318.

\begin{abstract}
Keywords

- hypertension

- postpartum

- remote monitoring

- telehealth

- blood pressure

- COVID-19

The transformation of our health care system in response to coronavirus disease 2019 (COVID-19) provides a unique opportunity to examine the use of telehealth for postpartum care. The postpartum period can pose significant risks and challenges, particularly for women with hypertensive disorders of pregnancy. Remote blood pressure monitoring has proven feasible and acceptable among women and providers but has not been widely implemented or researched. Early studies have identified improved outcomes with use of telehealth, including increased compliance with care and decreased disparity in hypertension follow-up. Preliminary data make a compelling case for remote monitoring as a promising treatment strategy to manage postpartum hypertension. Remote monitoring technology should be incorporated as a standard component for the comprehensive management of postpartum hypertension during COVID-19. As a consequence of the pandemic, we now have an opportunity to research the impact of postpartum remote blood pressure monitoring on maternal outcome and disparities within these outcomes.
\end{abstract}

The novel coronavirus disease 2019 (COVID-19) pandemic is rapidly increasing the use of telehealth and remote patient monitoring. To promote social distancing, health care providers are reducing nonessential outpatient visits and replacing face-to-face visits with virtual ones. Prior barriers to use these technologies are being addressed at the state and national levels as part of pandemic response. Given the concurrent crisis of increasing maternal mortality rates, postpartum women are an important population who stand to benefit from the innovative transformations in health care service delivery during COVID-19.

Postpartum follow-up is an essential component of pregnancy care, as half of all maternal deaths occur after delivery. ${ }^{1}$ Hypertensive disorders accounted for $6.9 \%$ of maternal deaths received

April 23, 2020

accepted

May 9, 2020
DOI https://doi.org/

10.1055/s-0040-1715169. ISSN 2157-6998.
Copyright $\odot 2020$ by Thieme Medical Publishers, Inc., 333 Seventh Avenue, New York, NY 10001, USA. Tel: +1(212) 760-0888.
License terms

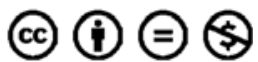


in the United States between 2011 and 2016, and more than $60 \%$ of deaths due to gestational hypertensive disease occur during the first 6 weeks postpartum. ${ }^{1}$ However, only $52 \%$ of women with severe pre-eclampsia attend their 6 -week postpartum follow-up, and of those who are able to access postpartum care, one in five is persistently hypertensive. ${ }^{2}$ Shifting from a strategy of in-person office visits to televisits in response to the COVID-19 pandemic may actually increase access to postpartum follow-up among women with hypertensive disorders of pregnancy.

The American College of Obstetricians and Gynecologists (ACOG) established guidelines for appropriate health care delivery during the COVID-19 pandemic, emphasizing virtual visits, remote patient monitoring, and mobile health care (grouped together as "telehealth"). ${ }^{3}$ However, implementation of these technologies is not widespread and evidence is still limited. With a nod to the adage, "necessity is the mother of invention," the need for social distancing in response to the COVID-19 pandemic may prove to be a catalyst for innovation in postpartum care. Any upsurge of remote monitoring for postpartum hypertension during COVID-19 must be accompanied by concurrent study of its broader public health impacts.

\section{Guidelines for Postpartum Blood Pressure Monitoring}

ACOG recommends blood pressure monitoring in the hospital during the first 72 hours after delivery, the duration of a typical postpartum stay. ${ }^{4-6}$ After discharge, a blood pressure check between 7 and 10 days postpartum at the hospital or a comparable outpatient facility is advised, prior to the routine visit at 6 weeks. ${ }^{4,6}$ While blood pressure goals for women with hypertensive disorders may require initiation of antihypertensive therapy, the overall approach to hypertension management in the postpartum period is varied in general, and the range of approaches in the time of COVID-19 remains unknown. ${ }^{4-6}$ Remote blood pressure monitoring can serve as a way to improve adherence to the above recommendations after delivery. A systematic review of telehealth in obstetrics and gynecology found that remote monitoring not only addressed barriers to care but also had particularly beneficial health outcomes for women with gestational diabetes and hypertension. ${ }^{7}$ Among postpartum women, remote monitoring can identify those with severe hypertension and by early initiation of antihypertensive therapy may reduce hospital readmissions and associated complications. ${ }^{8}$

\section{Current Evidence for Postpartum Remote Blood Pressure Monitoring}

Remote monitoring technologies have demonstrated promise for improving outcomes such as increased engagement with care, decreased hospital readmissions, and decreased use of antihypertensive medication in nonobstetric populations. However, more long-term follow-up data are lacking. Among postpartum women, data on both short-term and long-term outcomes are limited as well. Data from pilot studies indicate remote blood pressure monitoring is feasible and acceptable to patients and providers. Specifically, most women reported that they found the technologies easy to use, would recommend them to other women, and preferred this model of hypertension management to in-person clinic visits. ${ }^{8-10}$ One study found remote postpartum blood pressure monitoring increased in-person care engagement, with $88 \%$ of women who used remote monitoring attending their in-person 6-week postpartum visit, compared with a baseline attendance rate of $66 \%{ }^{10}$ Furthermore, $82 \%$ of patients expressed relief in knowing that a nurse was monitoring their blood pressure on a daily basis. ${ }^{10}$ In another study that coupled home blood pressure cuffs with text message reminders for remote postpartum blood pressure monitoring, $84 \%$ of participants were able to obtain at least one blood pressure reading at home within the first 10 days postpartum, consistent with ACOG guidelines, compared with 30 to $50 \%$ of patients receiving a single office-based blood pressure reading. ${ }^{11}$

Home telemonitoring following hypertensive pregnancies may additionally serve to reduce disparities in postpartum care. Black women are at higher risk of developing hypertensive disorders of pregnancy and at higher risk of death and poor pregnancy outcomes due to hypertension compared with nonblack peers. ${ }^{12,13}$ In a small trial, black women attended standard in-person visits significantly less than nonblack women ( 70 vs. $33 \%, p<0.001$ ), but when engaged in a virtual blood pressure monitoring program, both black and nonblack women demonstrated compliance rates of more than $90 \%{ }^{14}$

While this early literature in the postpartum population is promising, further study is needed to identify strategies to engage and retain participation in telehealth and to assess the long-term outcomes such as hospital readmissions, maternal mortality, and future cardiovascular health.

\section{Future Research Directions for Postpartum Remote Monitoring}

Scale-up of remote postpartum blood pressure monitoring during COVID-19 provides an important opportunity for research. Outstanding questions remain around potential limitations. For example, barriers in access to the technologies needed for these protocols may inadvertently contribute to inequities in care. Between 4 and $8 \%$ of women do not have access to smartphones, precluding any remote monitoring protocols that utilize smartphone apps. ${ }^{10,15}$ Furthermore, the women who do not have smartphone access are likely those who face significant challenges in accessing clinicbased care and follow-up, among other social determinants of health that put them at risk for poor health outcomes. Protocols such as the one described by Hirshberg et al that relied on text messaging only, ${ }^{11,14}$ rather than Bluetooth enabled smartphone apps, may be more feasible to implement equitably. To ensure that scale-up of this intervention does not inadvertently perpetuate inequities, rather than diminish them, data on these protocols in more diverse populations are also needed. Similarly, the majority of studies on remote blood pressure monitoring were conducted in English-speaking populations, and phone-accessible translation services and multilanguage text messaging options 
will be critical to establishing more robust evidence for deploying remote monitoring universally and equitably. ${ }^{8-11}$

The impact of changes in insurance coverage for telehealth and blood pressure cuffs during COVID-19 is also a critical area for research. Prior to the pandemic, limited insurance coverage during the postpartum period, as well as for telehealth visits, presented a major barrier to scaling up remote hypertension monitoring. Typically, telehealth appointments are not covered by health insurance in the same way as in-person appointments. Prior to COVID-19, only 19 states allowed Medicaid coverage of telehealth care, ${ }^{16}$ although Medicaid programs are rapidly expanding coverage for telehealth during the pandemic. ${ }^{17}$ Home blood pressure cuffs-typically difficult to obtain by Medicaid enrollees-are also becoming more available during COVID-19. ${ }^{18,19}$ However, despite these changes, a significant remaining limitation is that this coverage expires 60 days after delivery. ${ }^{20}$ As remote monitoring is more widely implemented during COVID-19, research is needed to examine the impact of telehealth and home blood pressure cuff Medicaid coverage on access to postpartum hypertension care as well as on maternal morbidity and mortality. Surveillance data on the impact of systemic changes due to COVID-19 may support future expansions of Medicaid coverage for postpartum women. ${ }^{21}$ Expansion of postpartum telehealth care and broader insurance coverage during this period may improve access not only to postpartum hypertension care but also to other critical services, including breastfeeding support, contraception counseling, and screening for postpartum depression.

\section{Opportunity within a Pandemic}

Even in the best of times, close postpartum follow-up of women with hypertensive disorders is a challenge. Today's acute need for physical distancing in response to the COVID19 pandemic poses even greater risk to this important component of pregnancy care. Remote blood pressure monitoring may mitigate some of this risk and preliminary data suggest high levels of patient satisfaction, increased patient engagement in postpartum care, and improved medication compliance. However, research is needed to assess the efficacy and impact of remote blood pressure monitoring in the postpartum period on clinical outcomes including hospital readmissions, short-term medical complications of hypertension, and long-term cardiovascular health for patients with hypertensive disease of pregnancy.

While the imperative to roll out telehealth care for postpartum hypertension exists, several barriers must be overcome including affordable home devices, accessible education on blood pressure cuff use in multiple languages, and an effective virtual clinical protocol. Closely evaluating the clinical outcomes and health care disparities that follow the rapid implementation of home blood pressure monitoring in the postpartum period during COVID-19 will provide valuable lessons for research and clinical care after the pandemic. The COVID-19 pandemic presents a rapid care redesign opportunity to establish telehealth protocols that if carefully monitored for equity and quality may improve care for postpartum women in the long term.

\section{Funding}

Risk Management Foundation of Harvard Medical Institutions, Inc. (nonprofit) 2018A016340.

Conflict of Interest

None declared.

\section{References}

1 Petersen EE, Davis NL, Goodman D, et al. Vital signs: pregnancyrelated deaths, United States, 2011-2015, and strategies for prevention, 13 States, 2013-2017. MMWR Morb Mortal Wkly Rep 2019;68(18):423-429

2 Levine LD, Nkonde-Price C, Limaye M, Srinivas SK. Factors associated with postpartum follow-up and persistent hypertension among women with severe preeclampsia. J Perinatol 2016;36 (12):1079-1082

3 Presidential Task Force on Telehealth. Implementing telehealth in practice. Obstet Gynecol 2020;135(02):e73-e79

4 Hauspurg A, Countouris ME, Catov JM. Hypertensive disorders of pregnancy and future maternal health: how can the evidence guide postpartum management? Curr Hypertens Rep 2019;21 (12):96

5 ACOG Practice Bulletin No. 202: Gestational Hypertension and Preeclampsia. Obstet Gynecol 2019;133(01):211-214

6 Cairns AE, Pealing L, Duffy JMN, et al. Postpartum management of hypertensive disorders of pregnancy: a systematic review. BMJ Open 2017;7(11):e018696

7 DeNicola N, Grossman D, Marko K, et al. Telehealth interventions to improve obstetric and gynecologic health outcomes: a systematic review. Obstet Gynecol 2020;135(02):371-382

8 Hoppe KK, Williams M, Thomas N, et al. Telehealth with remote blood pressure monitoring for postpartum hypertension: a prospective single-cohort feasibility study. Pregnancy Hypertens 2019;15:171-176

9 Rhoads SJ, Serrano CI, Lynch CE, et al. Exploring implementation of m-health monitoring in postpartum women with hypertension. Telemed J E Health 2017;23(10):833-841

10 Hauspurg A, Lemon LS, Quinn BA, et al. A postpartum remote hypertension monitoring protocol implemented at the hospital level. Obstet Gynecol 2019;134(04):685-691

11 Hirshberg A, Downes K, Srinivas S. Comparing standard officebased follow-up with text-based remote monitoring in the management of postpartum hypertension: a randomised clinical trial. BMJ Qual Saf 2018;27(11):871-877

12 Breathett K, Muhlestein D, Foraker R, Gulati M. Differences in preeclampsia rates between African American and Caucasian women: trends from the National Hospital Discharge Survey. J Womens Health (Larchmt) 2014;23(11):886-893

13 Ananth CV, Keyes KM, Wapner RJ. Pre-eclampsia rates in the United States, 1980-2010: age-period-cohort analysis. BMJ 2013;347:f6564

14 Hirshberg A, Sammel MD, Srinivas SK. Text message remote monitoring reduced racial disparities in postpartum blood pressure ascertainment. Am J Obstet Gynecol 2019;221(03):283-285

15 Pew Research Center. Demographics of mobile device ownership and adoption in the United States. Pew Research Center: Internet, Science \& Tech. Available at: https://www.pewresearch.org/internet/fact-sheet/mobile/. Accessed January 24, 2020

16 Weigel G, Frederiksen B. February 26 URP, 2020. Telemedicine and pregnancy care. The Henry J Kaiser Family Foundation. February 2020. Available at: https://www.kff.org/womens-health-policy/issuebrief/telemedicine-and-pregnancy-care/. Accessed March 27, 2020 
e318 Remote Monitoring for Postpartum Hypertension During COVID-19 Sawyer et al.

17 Medicaid.gov. COVID-19 frequently asked questions (FAQs) for state Medicaid and Children's Health Insurance Program (CHIP) agencies. Available at: https://www.medicaid.gov/state-resource-center/Downloads/covid-19-faqs.pdf. Accessed April 22, 2020

18 Bluth R. When getting a blood pressure cuff takes all day. NPR.org. February 2016. Available at: https://www.npr.org/sections/healthshots/2016/02/18/467209573/when-getting-a-blood-pressure-cuff -takes-all-day. Accessed April 22, 2020

19 NC Medicaid. SPECIAL BULLETIN COVID-19 \#29: Coverage for automatic blood pressure monitors: temporary flexibilities effective
March 30, 2020. Available at: https://medicaid.ncdhhs.gov/blog/ 2020/03/31/special-bulletin-covid-19-29-coverage-automaticblood-pressure-monitors-temporary. Accessed April 22, 2020

20 Eckert E. It's past time to provide continuous Medicaid coverage for one year postpartum | Health Affairs. February 2020. Available at: https://www.healthaffairs.org/do/10.1377/hblog20200203.639479 /full/. Accessed April 22, 2020

21 Lackland DT. Racial differences in hypertension: implications for high blood pressure management. Am J Med Sci 2014;348(02): 135-138 